\title{
Design and Analysis of an Adaptive Boost Converter for Piezoelectric Energy Harvester
}

\author{
Kunpeng Wang ${ }^{\mathrm{a}}$, Dazheng $\mathrm{Xu}^{\mathrm{b}}$ and Mingjie Guan ${ }^{\mathrm{c}}$ \\ School of Aerospace Engineering, Xiamen University, Xiamen 361005, China \\ akpwang3@xmu.edu.cn, bdzhxu2@xmu.edu.cn, ${ }^{\mathrm{c}} \mathrm{mjguan} @ x \mathrm{xmu} . e d u . c n$
}

Keywords: Piezoelectric energy harvesting, boost converter, efficiency, self-start.

\begin{abstract}
Using piezoelectric elements to harvest energy from ambient vibrations has been of great interest over the past few years. This paper presents a two-stage adaptive boost converter with low-voltage starter for low voltage piezoelectric energy harvester. The adaptive technique can deal with a range of input voltages from the excited piezoelectric generator for required output voltages. The parameters of the converter including inductance, switching frequency, duty cycles and the input/output voltage are studied for better conversion efficiencies. The simulation results show that a two-stage adaptive boost converter can achieve a high efficiency of $92.3 \%$ for low voltage piezoelectric harvester to charge an energy storage device with higher voltage.
\end{abstract}

\section{Introduction}

Wireless sensors have being adopted in monitoring human health, infrastructures, smart buildings and remote areas. A critical component of the wireless sensor is the power supply. Traditional batteries are usually used to power wireless sensors. But in many applications, replacing batteries, due to their limited lifetime, is inconvenient. In recent years, harvesting energy from the environment to power the wireless sensors is attracting more and more attentions. The environmental energy sources for energy harvesting can be solar, thermal, vibration, and RF energy as well as wind. While there are several options for obtaining the ambient energy, piezoelectric transducers have been of great interest and promise [1-6]. Many adaptive piezoelectric harvesting circuits are built for the piezoelectric energy harvesting system. Most of them are based on buck or step-down converter schemes, for example the commercial solution of LTC3588-1[7]. However for many applications the output voltage of the piezoelectric harvester is too low for a buck converting scheme. As shown in [1, 2], the peak output voltages for acoustic energy generators were below $0.105 \mathrm{~V}$ and $1.5 \mathrm{~V}$ respectively. The peaks of voltage output of acetone/MEK film in [3] only reached $2 \mathrm{~V}$. Piezoelectric voltage outputs from jaw movements in [4] were lower than $1 \mathrm{~V}$. Therefore, it is demonstrated that the piezoelectric energy harvesting circuit should be able to deal with a range of low voltage inputs. In this paper, our target is to study the harvesting scheme for low voltage piezoelectric generator with peak open-circuit voltage ranging from $0.5 \mathrm{~V}$ to $2 \mathrm{~V}$.

Boost converter have been studied for thermoelectric energy harvesting [8, 9]. However, piezoelectric generator is different from the thermoelectric generator in high internal capacitive resistance and alternating source. Therefore, the conventional dc-dc boost scheme for thermoelectric generator should be modified and developed towards piezoelectric generator. In this paper, a two-stage adaptive DC-DC boost converter for low voltage piezoelectric generator is presented. The adaptive technique that adjusts the switching frequency and duty cycles of the boost converter according to system variations is demonstrated.

\section{Theory}

\subsection{Piezoelectric Generator Model and One-stage Energy Harvesting Circuit}

A harmonic vibrating piezoelectric element can be modeled as a sinusoidal current source $i_{p}$ in parallel with its internal capacitance $C_{p}$ as shown in Figure 1. 
where $I_{p}$ and $\omega$ are the amplitude and frequency of the current source, respectively. The amplitude of the current $I_{p}$ varies with the mechanical excitation level of the piezoelectric element. But it is assumed to be relatively unchanged regardless of the external harvesting circuit [10]. A basic harvesting circuit is a one-stage harvesting circuit. Because the current source is alternating, an AC-DC rectifier is needed. A diode bridge rectifier is generally used as the AC-DC rectifier. As developed by Ottman et al. [10], there exists an optimal rectifier voltage $V_{\text {rect_opt }}$ to harvest the power. The voltage is related to the current amplitude $I_{p}$, frequency $\omega$, and the capacitance of the piezoelectric element $C_{p}$ by $V_{\text {rect_opt }}=I_{p} /\left(2 \omega C_{p}\right)$. The optimal rectifier voltage $V_{\text {rect_opt }}$ is one-half the amplitude of the peak open-circuit voltage of the piezoelectric element $V_{\text {oc }}$. The power harvested at the optimal voltage $V_{\text {rect_opt }}$ is

$$
P=V_{\text {rect_opt }} \overline{I_{\text {esd }}}=V_{\text {rect_opt }} \cdot \frac{2}{\pi}\left(I_{p}-\omega V_{\text {rect_opt }} C_{p}\right)
$$

where $\overline{I_{\text {esd }}}$ represents the average current into the energy storage device.

\subsection{Two-stage Energy Harvesting Circuit}

To harvest the power from varying exciting sources, another scheme uses a two-stage harvesting circuit. A two-stage harvesting scheme can be generalized as shown in Figure 1. The power from the piezoelectric element is firstly rectified to the temporary storage with a capacitor $C_{i}$ whose voltage $V_{\text {in }}$ is kept at the optimal rectifier voltage $V_{\text {rect_opt }}$. The input power from the piezoelectric element to the capacitor $C_{i}$ is then transferred to the energy storage device through a DC-DC converter.

The self-start of the dc-dc converter must be supported by a high voltage from a low-voltage starter. The piezoelectric generator output is connected to a low-voltage starter through switch $S_{1}$. When the output voltage of the low-voltage starter is high enough for the controller and boost converter, the piezoelectric generator output is switched to the boost converter. To implement the adaptive system, the open-circuit voltage of the PEG is measured periodically. The harvesting circuit is periodically disconnected from the piezoelectric element by the switch $S_{2}$ and the open-circuit voltage is measured during the short disconnection period. The tracking technique for the adaptive scheme is implemented by adjusting the switching frequency and duty cycles of the boost converter.

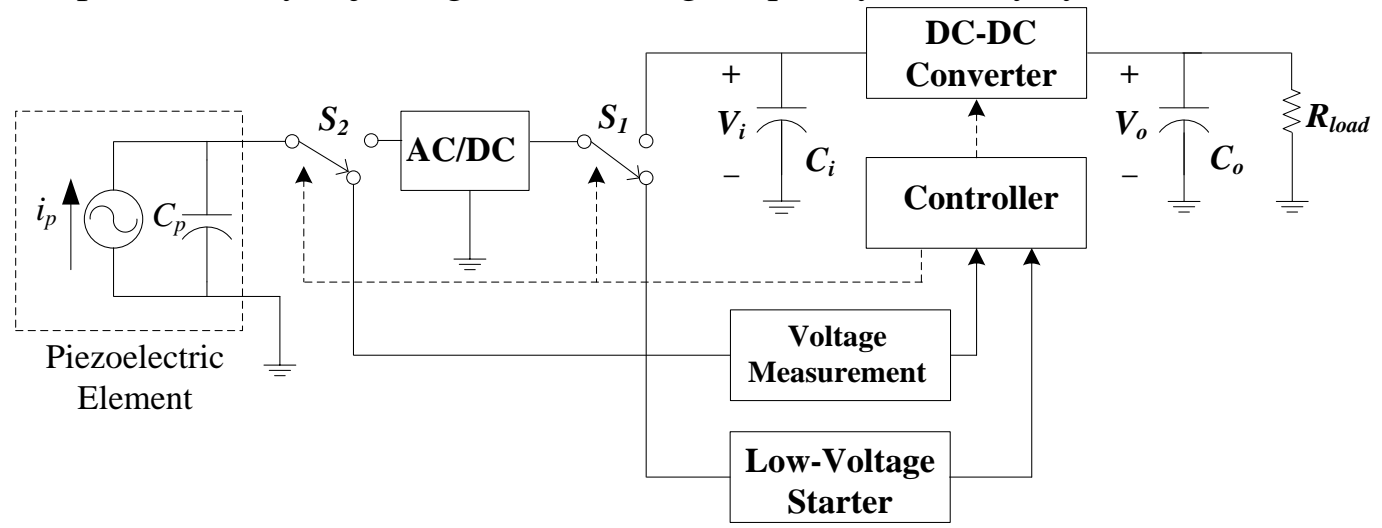

Fig. 1 Two-stage piezoelectric energy harvesting scheme

\subsection{DC-DC Converter Circuit}

The DC-DC converter used in the two-stage harvesting scheme is shown in Figure 2. The converter includes two voltage-controlled on-off switches $G_{1}$ and $G_{2}$, and an inductor $L$. Discontinuous conduction mode (DCM) is considered in the switching converter implemented in low-power energy harvesting circuits.

The operating procedures and waveforms of the converter in DCM mode are shown in Figures $2 \& 3$, respectively. At the beginning, the capacitor $C_{i}$ is charged. When the voltage of the capacitor 


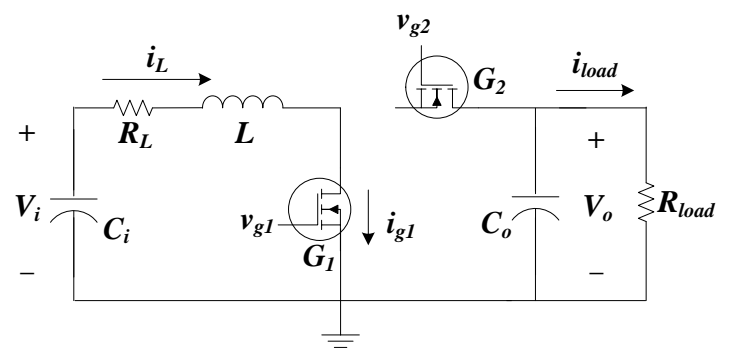

(a)

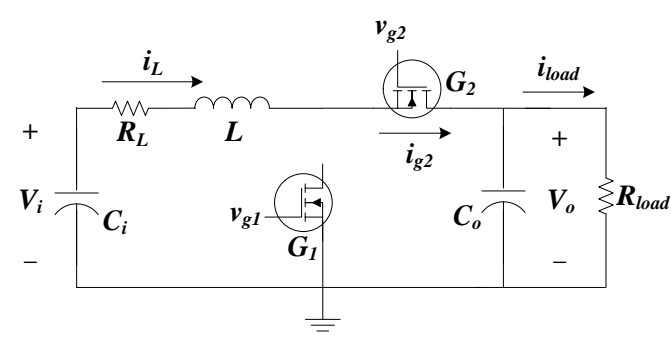

(b)

Fig. 2 Converter operations (a) switch G1 is "on" and switch G2 is "off”

(b) switch G1 is "off” and switch G2 is "on”

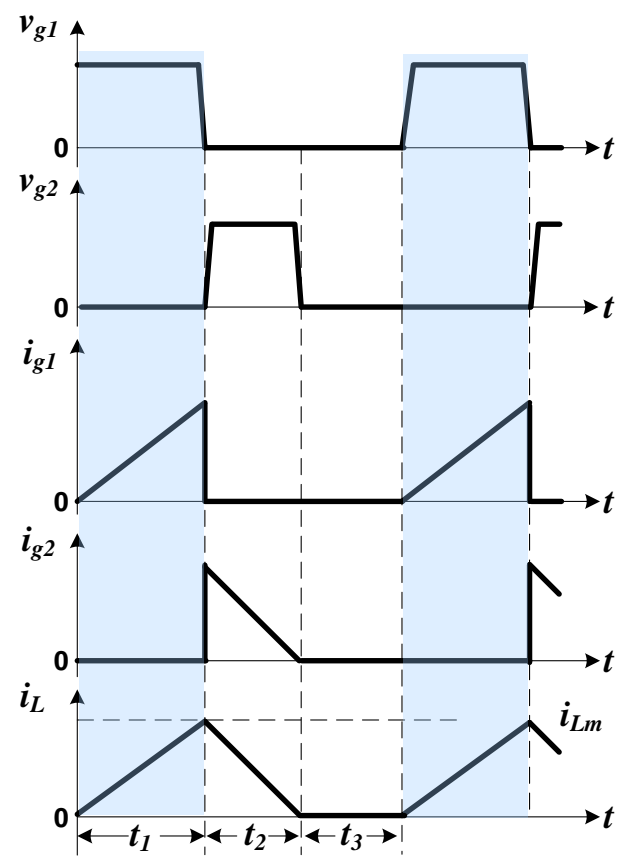

Fig. 3 Waveforms of the voltages and currents in the converter

$C_{i}$ reaches the optimal voltage $V_{\text {rect_opt }}$, the converter starts working. The switches are controlled to switch on or off in order to keep the voltage across the capacitor $C_{i}$ at an optimal value. When switch $G_{1}$ is "on" and switch $G_{2}$ is "off" (see Figure 2(a)), due to the presence of the inductor $L$, the current $i_{L}$ will increase gradually from zero. When the control voltage $v_{g 1}$ falls down and $v_{q 2}$ rises up, the switch $G_{1}$ is turned “off” and switch $G_{2}$ is “on" (see Figure 2(b)). The current $i_{L}$ will decrease to zero in the interval $t_{2}$. As shown in Figure 3 , in the internal $t_{1}$, the current linearly increases with time, we have

$$
\frac{\Delta i_{L}}{t_{1}}=\frac{V_{i}-i_{L} R_{L}-V_{o n 1}}{L}=\frac{i_{L m}}{t_{1}}
$$

where $L$ is the inductance of the inductor; $i_{L m}$ is the maximum current reached; $V_{\text {on1 }}$ is the voltage drop of the switch when switch $G_{1}$ is "on".

In order to keep the voltage $V_{i}$ at an optimal value, the average current $\overline{i_{L}}$ should be equal to the average current $\overline{I_{\text {esd }}}$ in Equation (2). To make it simple, the voltage drop on the internal resistance of the inductor $i_{L} R_{L}$ is neglected. We have

$$
\frac{V_{i}-V_{\text {on } 1}}{L}=\frac{i_{L m}}{t_{1}}=\frac{2 \bar{i}_{L}}{t_{1}} \times \frac{t_{1}+t_{2}+t_{3}}{t_{1}+t_{2}}=\frac{2 \bar{I}_{\text {rect_opt }}}{t_{1}} \times \frac{t_{1}+t_{2}+t_{3}}{t_{1}+t_{2}}
$$

Assuming $t_{3}=(\beta-1)\left(t_{1}+t_{2}\right)$, we have 
$t_{1}=2 \beta L \times \frac{\bar{I}_{\text {rect_opt }}}{V_{i}-V_{\text {on } 1}}$

In the interval $t_{2}$, the current $i_{L}$ will decrease linearly from $i_{L m}$ to zero with time. We have

$t_{2}=2 \beta L \times \frac{\bar{I}_{\text {rect_opt }}}{V_{o}+V_{\text {on } 2}}$

Thus, the efficiency of the converter can be calculated as

$\eta_{c}=\frac{\overline{i_{\text {load }}} \times V_{o}}{\overline{i_{L}} \times V_{i}}=\frac{V_{i}-V_{\text {on } 1}}{V_{o}-V_{\text {on } 1}+V_{\text {on } 2}} \times \frac{V_{o}}{V_{i}}$

From Equation (7), it can be found that the efficiency is related to $V_{o}, V_{i}, V_{\text {on1 }}$ and $V_{\text {on2 }}$.

The switching period $T_{s}$ is

$T_{s}=t_{1}+t_{2}+t_{3}=2 \beta \bar{I}_{\text {esd }} \times\left(\frac{V_{i}}{V_{i}-V_{\text {on } 1}}+\frac{V_{i}}{V_{o}-V_{i}+V_{\text {on } 2}}\right)$

The duty cycle of the PWM control signal of the gate $G_{1}$ is

$D C_{1}=\frac{t_{1}}{t_{1}+t_{2}+t_{3}}$

The duty cycle of the PWM control signal of the gate $G_{2}$ is

$D C_{2}=\frac{t_{2}}{t_{1}+t_{2}+t_{3}}$

A predetermined load resistance $R_{\text {load }}$ is

$R_{\text {load }}=\frac{V_{o}^{2}}{\eta_{c} V_{i} \bar{I}_{\text {esd }}}$

\section{Simulation and results}

Simulations were carried out for the verification on the two-stage harvesting scheme. The circuit for the two-stage energy harvesting scheme built in PSPICE is shown in Figure 4 . The frequency $f_{p}$ of the current source $i_{p}$ is set to $50 \mathrm{~Hz}$, and the internal capacitance $C_{p}$ is $35.7 \mathrm{nF}$ in the circuit. Other parameters are as follows: $R_{p}, R_{s}$ represent the internal resistive loss of the piezoelectric element, which are $5 \mathrm{M} \Omega$ and $1 \Omega$, respectively (Guan and Liao [11]); the inductance $L$ is $1 \mathrm{mH} ; R_{L}(1.12 \Omega)$ is the internal resistance of the inductor $L$; the input capacitor $C_{i}$ is $1 \mu F$; the output capacitor $C_{o}$ is $1 \mu F$; $\mathrm{G}_{1}$ and $\mathrm{G}_{2}$ are standard MOSFETs (model BSS138/FAI); D1 D4 are Schottky diodes with $100 \mathrm{mV}$ turn-on voltage (model D1N5817); $v_{g 1}$ and $v_{g 2}$ are the PWM control signals. The low level, high level, rise time, and fall time of control voltages $v_{g 1}$ and $v_{g 2}$ are set as $0 V, 3 V, 0.1 \mu s$, and $0.1 \mu s$, respectively. The square wave starts to be applied to the switches when $V_{\text {in }}$ reaches the optimal value. Before that moment, the switch "SW" is kept at "off" state. Voltage drops $V_{\text {on1 }}$ and $V_{\text {on2 }}$ when the gates G1 and G2 are "on” state are both $0.03 \mathrm{~V}$, which are obtained from the component datasheet.

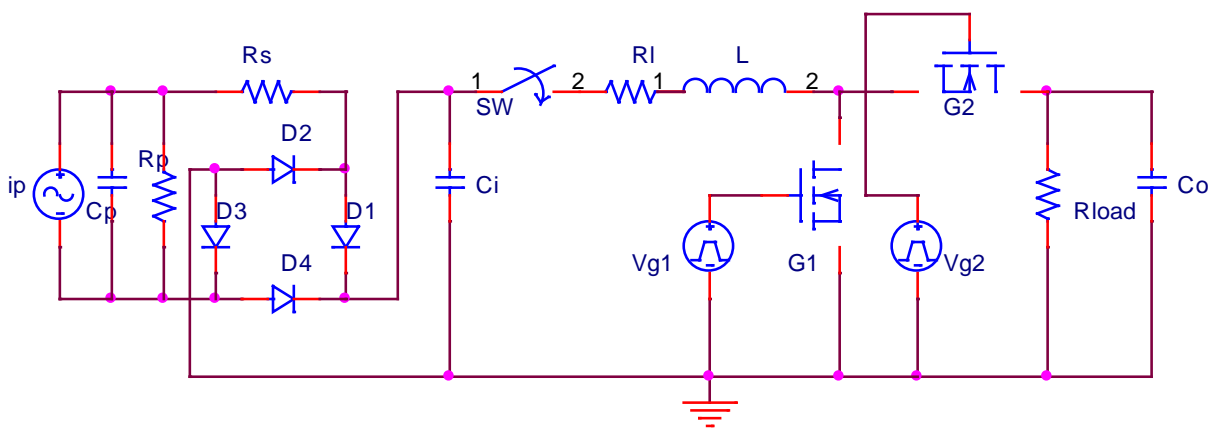

Fig. 4 Two-stage energy harvesting circuit built in PSPICE 
Simulation is carried out to validate the efficiency equation for different input voltage. The amplitude of the current source varies from $5.61 \mu \mathrm{A}$ to $22.43 \mu \mathrm{A}$, corresponding to the peak open-circuit voltage from $0.5 \mathrm{~V}$ to $2 \mathrm{~V}$. For each peak open-circuit voltage $V_{o c}$, the rectifier voltage is kept at the optimal value from $0.25 \mathrm{~V}$ to $1 \mathrm{~V}$ by modulating the frequency and duty cycles of the control voltages based on Equations (8-10). The target output voltage is set $1.5 \mathrm{~V}$. From Equation (11), the load resistor $R_{\text {load }}$ is set for each peak open-circuit voltage. Based on Equation (7), the theoretical efficiencies of the converter are obtained as shown in the solid curve in Figure 5. The efficiency can also be calculated from the PSPICE simulation results as shown in Figure 5.

It can be seen from Figure 5 that those two curves match well. Therefore, the efficiency expressed in Equation (7) is verified by the simulation. The efficiency curve in simulation is below the efficiency curve in theory because in the theoretical calculation the resistive loss of the inductor $L$ is neglected but in simulation not.

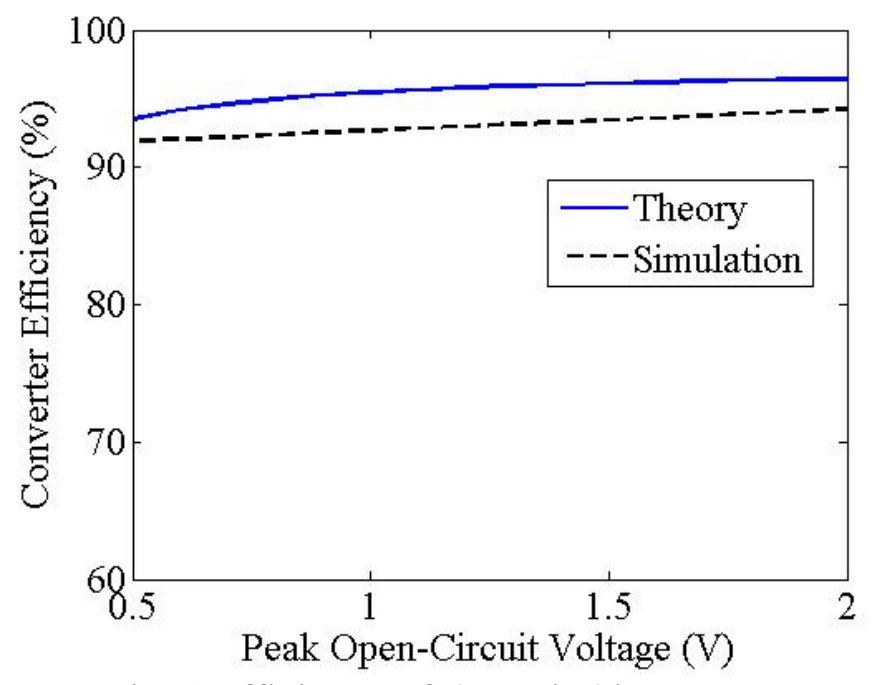

Fig. 5 Efficiency of the switching converter

\section{Conclusions and discussions}

An adaptive dc-dc boost converter with low-voltage starter for low voltage piezoelectric generators was designed and analyzed in this paper. The adaptive scheme dynamically controls the switching frequency and duty cycles by sensing the open-circuit voltage of the piezoelectric generators and extracts the maximum power from the piezoelectric generators. The switch control circuit finely controls the on-off switches at the optimal time to fulfill a discontinuous conductive mode.

Expressions of the optimal switching frequency, the duty cycles and the efficiency of the converter were derived. It is shown that the converter efficiency is related to several parameters including the voltage drop of the switches, the input voltage and the output voltage. The efficiency could be possibly improved by decreasing the voltage drops of the switches. Simulation results show that the adaptive boost converter can achieve a high efficiency of 92.3\% 94.8\% for piezoelectric harvester with open-circuit voltage of $0.5 \mathrm{~V} \sim 2 \mathrm{~V}$ to charge a $1.6 \mathrm{~V}$ storage voltage.

The controller in the DC-DC converter will consume a certain part of collected energy, which will cancel out part of the harvested energy. However, there could be a possible way to apply the two-stage scheme on the piezoelectric energy harvesting circuit, provided that the consumed power in the DC-DC converter was reduced to an acceptable level.

\section{Acknowledgments}

The work described in this paper was supported by a grant from the China Science Foundation (project no. 11202176) and a grand from China Scholarship Council. 


\section{References}

[1]. Li B, Laviage AJ, You JH, et al. Harvesting low-frequency acoustic energy using multiple PVDF beam arrays in quarter-wavelength acoustic resonator. Appl Acoust. Vol. 74 (2013), p. 1271-1278.

[2]. Li B, You JH, Kim YJ. Low frequency acoustic energy harvesting using PZT piezoelectric plates in a straight tube resonator. Smart Mater Struct. Vol. 22 (2013), p. 055013.

[3]. Chen D, Sharma T, Zhang JX. Mesoporous surface control of PVDF thin films for enhanced piezoelectric energy generation. Sensor Actuat A-Phys. Vol. 216(2014), p.196-201.

[4]. Delnavaz A and Voix J. Flexible piezoelectric energy harvesting from jaw movements. Smart Mater Struct. Vol. 23 (2014), p. 105020.

[5]. Xu TB, Siochi EJ, Kang JH, et al. Energy harvesting using a PZT ceramic multilayer stack. Smart Mater Struct. Vol. 22 (2013), p. 065015.

[6]. He XF and Gao J: Wind energy harvesting based on flow-induced-vibration and impact. Microelectron Eng. Vol. 111 (2013), p. 82-86.

[7]. Linear Technology, “Nanopower Energy Harvesting Power Supply,” LTC3588-1 datasheet.

[8]. Carlson EJ, Strunz K, Otis BP. A $20 \mathrm{mV}$ input boost converter with efficient digital control for thermoelectric energy harvesting. IEEE J Solid-St Circ. Vol. 45 (2010) No. 4, p. 741-750.

[9]. Teh YK and Mok KT. Design of transformer-based boost converter for high internal resistance energy harvesting sources with $21 \mathrm{mV}$ self-startup voltage and 74\% power efficiency. IEEE J Solid-St Circ. Vol. 49 (2014) No. 11, p. 2694-2704.

[10]. Ottman GK, Hofmann HF, Bhatt AC, et al. Adaptive piezoelectric energy harvesting circuit for wireless remote power supply. IEEE T Power Electr. Vol. 17 (2002) No. 5, p. 1-8.

[11]. Guan MJ and Liao WH. On the equivalent circuit models of piezoelectric ceramics. Ferroelectrics. Vol. 386 (2009) No. 1, p. 77-87. 